\title{
Conceptual contributions to the determination of the curvature in Engineering courses
}

\section{Aportaciones conceptuales sobre la curvatura en los estudios en Ingeniería}

VÁZQUEZ-GONZÁLEZ, Benjamín†*, JIMÉNEZ-RABIELA, Homero, BRAVO-ACOSTA, Adrian Gustavo and QUINTANA-DIAZ, María Berenice Guadalupe

Universidad Autónoma Metropolitana, Unidad Azcapotzalco, División de Ciencias Básicas e Ingeniería, Departamento de Energía

ID $1^{\text {st }}$ Author: Benjamín, Vázquez-González / ORC ID: 0000-0002-9030-5662, Researcher ID Thomson: S-2417-2018, CVU CONACYT ID: 25749

ID $1^{\text {st }}$ Coauthor: Homero, Jiménez-Rabiela / Researcher ID Thomson: S-2299-2018, CVU CONACYT ID: 123386

ID $2^{\text {nd }}$ Coauthor: Adrian Gustavo, Bravo-Acosta / ORC ID: 0000-0001-57975317, Researcher ID Thomson: 2272-2018, CVU CONACYT ID: 334391

ID $3^{\text {rd }}$ Coauthor: María Berenice Guadalupe, Quintana-Diaz / ORC ID: 0000-0002-4703-1934, CVU CONACYT ID: 1015523

DOI: 10.35429/JMQM.2019.4.3.9.19

Received March 28, 2019; Accepted June 10, 2019

\begin{abstract}
Differential geometry began with the study of the characteristics of planar curves, then the behavior of the curves in space was analyzed, which led to the postulates of Frenet, and hence differential geometry evolved due to the contributions of Gauss. At the highly specialized undergraduate courses, most of the literature presents this topic based on definitions, which can be understood with some difficulty by both students and even some teachers. This work presents a detailed description of the terms defined in the concept of curvature. It is of great importance that students from engineering courses understand this concept with certainty and confidence, because it will allow perceiving abstract terms, such as radius of curvature, osculating circle, normal vector; thus, they will have complete handling in the basic description of the movement of bodies. Some examples are presented.
\end{abstract}

Education, Osculating circle, Curvature radio

\begin{abstract}
Resumen
La geometría diferencial se inició con el estudio de las características de las curvas planas, posteriormente se estudió el comportamiento de las curvas en el espacio, lo que llevó a los postulados de Frenet, y de ahí en adelante la geometría diferencial evolucionó grandemente, gracias a las aportaciones de Gauss. En los cursos de cálculo a nivel superior la mayor parte de la bibliografía presenta este tema a base de definiciones, que pueden comprenderse con un poco de dificultad tanto por parte de alumnos e inclusive de algunos docentes. En este trabajo se presenta una descripción detallada de las definiciones que se concretan en el concepto de la curvatura. La importancia de que los estudiantes de los cursos de ingeniería dominen con certeza este concepto es de gran valor, porque les permitirá percibir con claridad términos abstractos, tales como radio de curvatura, circulo osculatriz, vector normal, por ejemplo, y dominando estos términos se tendrá un manejo completo en la descripción básica del movimiento de cuerpos. Se presentan algunos ejemplos.
\end{abstract}

Educación, Circulo osculatriz, Radio de curvatura

Citation: VÁZQUEZ-GONZÁLEZ, Benjamín, JIMÉNEZ-RABIELA, Homero, BRAVO-ACOSTA, Adrian Gustavo and QUINTANA-DIAZ, María Berenice Guadalupe. Conceptual contributions to the determination of the curvature in Engineering courses. Journal-Mathematical and Quantitative Methods. 2019. 3-4: 9-19

\footnotetext{
* Correspondence to Author (email: bvg@correo.azc.uam.mx)

$\dagger$ Researcher contributing first author
} 


\section{Introduction}

Many areas of engineering use the geometric concept of curvature. In the study of bending in the strength of materials, the analytical definition of curvature is used to establish the differential equation of the elastic line, see for example Byras and Snyder. The determination of the Laplace-Young equation, as shown by Kralchevsky and Nagayama as well as Canoglu, in fluid mechanics requires the definition of the radius of curvature. In mechanics and in particular, in kinematics, the definition of curvature is fundamental to describe the curved movement of a body, as shown by Shigley and Uicker, where the velocity vector can change both its magnitude and its direction. However, in mechanics, this concept is also required as the basis for the study of the kinematics of mechanisms such as gears, as in Griffis.

The recent technological advances require a permanent evaluation of the technological advances, see Tenbergen, which forces a permanent evaluation of the teaching and study of mathematics was pointed out at the time by Klein, as revised in G. Kaiser, this has transcended in subsequent research, in particular towards the study of the curvature Gual-Arnau et. al. Klein makes particular reference to the concept of the curvature Guha, M, where it is quoted, "Everyone knows what a curve is, until he has studied enough mathematics to become confused through the countless number of possible exceptions." This shows the complexity of the subject, although this expression was published between the 19th and 20th century, the study of curvature is still in force.

Currently, it is proposed to study new types of curvature Gual-Arnau et al., but based on the concepts with which it was initially proposed. In the deduction of the equations mentioned above, the analytical definition of curvature is taken, considering that this concept is clear. However, this is not always the case, for example, when deducing the Laplace-Young equation, from fluid mechanics, in the study of capillarity, as in Adamson and Gast. It is interesting to observe how during the development one starts from the calculation of the work done on a moving surface, and the work must be determined using the difference in pressure.
Which leads to the calculation of the geometry of the surface area on which the work is done and this, in turn, requires knowledge of the radii of curvature of the surfaces that involve the displacement. The deduction of the LaplaceYoung equation is simplified, thanks to the previous existence of the expression of the curvature calculation. In this work, a detailed explanation of the expressions that make up the definition of curvature is given. The current bibliography and even the specific bibliography, such as the one cited above, present a series of definitions, which in general are not clear in their description. Hence, it becomes essential, in terms of engineering education, to have clarity about the meaning and significance of the concept of curvature.

In the following section, the definitions and concepts that are used to define the concept of curvature are presented. These are accompanied by explanations that give meaning to the analytical expressions that, in general, the bibliography does not present. This is one of the didactic contributions of this work. Section three illustrates the relationship between the radius of curvature and curvature; section four presents a demonstration of the uniqueness of the osculatrix circle. Finally, in section five, the conclusions are presented.

\section{Geometric elements of the curvature}

Historically it is known that it was Euler who formalized the study of curvature. However, Leibnitz and Newton had addressed the problem by making some approaches according to Montiel and Ros although Bardini and Gianella mention how Kästner too defined curvature at a point as the limit of the quotient between the angle $\Delta \theta$ and the arc length $\Delta \mathrm{s}$, as the two points that define the arc length approach, where the angle $\Delta \theta$ is defined by the difference in direction between the tangents existing between the points of the arc length. This definition appears again in the literature on the subject, which is quite extensive and systematically presents the definition of curvature, see for example Anton and Leithold. This systematic view in the courses corresponding to the development of the topic can be scarce in its appreciation. Here these concepts are taken up again. The definition of curvature is based on the existence of the vectors tangent to a curve and their variations on the same curve. 
Consider the following figure,

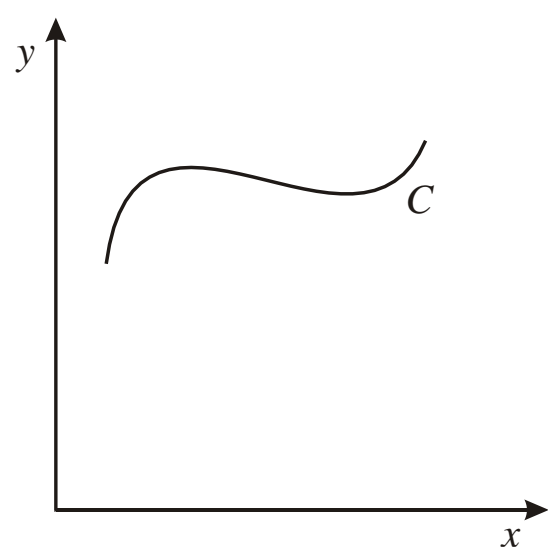

Figure 1 The characteristics of a flat $\mathrm{C}$ curve can be determined by placing the curve on a coordinated system

In the study of the structure of the curves, there are several ways to represent a $\mathrm{C}$ curve. It can have a functional expression of the type $\mathrm{y}=\mathrm{f}(\mathrm{x})$ or a parametric representation of the type $r=r(t)$, where $t$ is a scalar and $r$ is a vector.

The vector representation allows us to quantify in a direct way the variations of the curve, that is to say, the changes that appear just by increasing the $t$ parameter, this can also be achieved in a coordinated system that helps to locate each point of the curve in a vectorial way. In a vector representation of a curve, the parameter that defines it is not presented together with the path; it is necessary to consider that the parameter can be measured on a scale parallel to the coordinated system. Figure 2 shows a curve and the two possible graphical representations. Note that these representations are not in conflict.

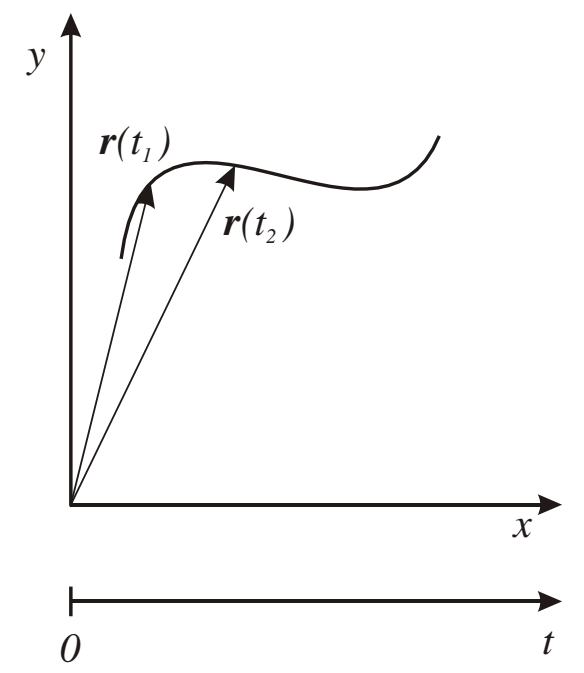

Figure 2 A curve can be represented by a functional relationship $\mathrm{y}=\mathrm{f}(\mathrm{x})$ or $\mathrm{r}=\mathrm{r}(\mathrm{t})$ in the same coordinate system, in the vector case the curve can be accompanied by its parameter $\mathrm{t}$
A fundamental characteristic of curves is the tangent lines. A vector representation of the tangent lines is provided by the tangent vector and the unit tangent vector. Figure 3 shows the variation of the position vector that describes the curve, as the parameter $t$ is increased.

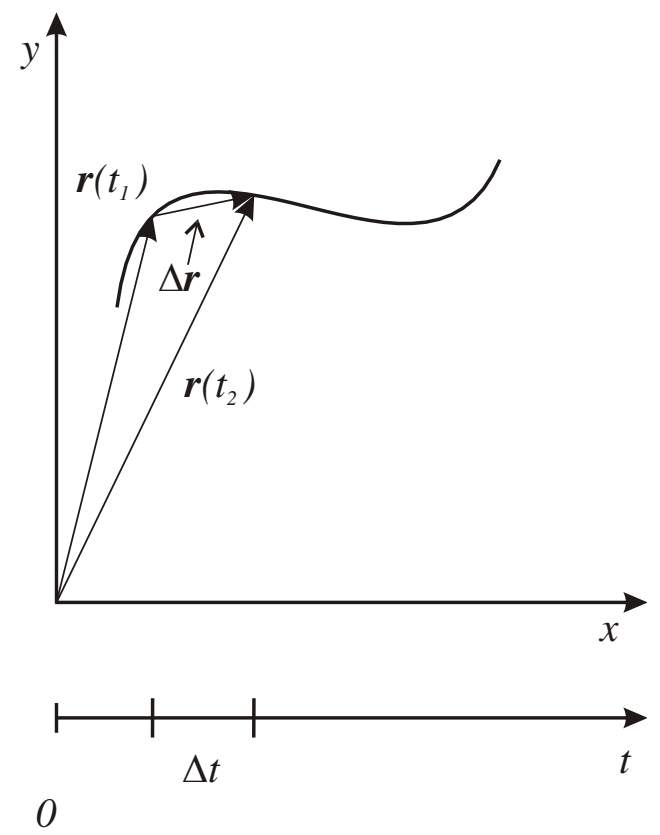

Figure 3 Vectorial variations describing the $\mathrm{C}$ curve, as the $t$ parameter is increased

The vector tangent to a curve is then determined using the vector representation. The definition of a derivative of a vector function allows the characteristics of the vector tangent to be observed.

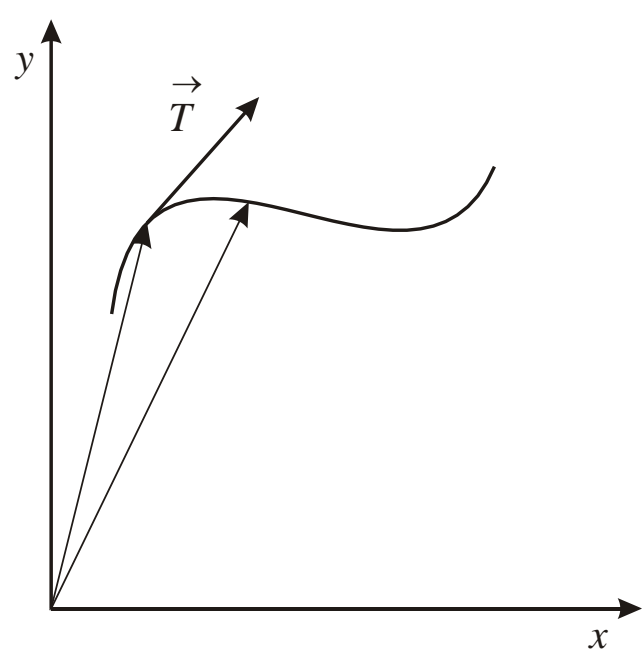

Figure 4 Determination of the tangent vector $\mathrm{T}=\mathrm{T}^{\rightarrow}$ to a curve and its graphic representation

Note that the tangent vector has been obtained more than the tangent line

$\vec{T}=\lim _{\Delta t \rightarrow 0} \frac{\Delta r}{\Delta t}=\frac{d r}{d t}=\boldsymbol{r}^{\prime}(t)$ 
The process of limit calculation is the primary tool that allows capturing the geometric characteristics of a curve. Note that in the evaluation of moving the parameter $\Delta t$ towards zero, i.e., $t_{2} \rightarrow t_{1}$, the vector $\Delta \boldsymbol{r}$, coincides at a point with the curve and being a vector that coincides with the curve, it is tangent and provides a measure of the change of the curve with respect to the parameter $t$. In a straight line, the measure is constant.

What has been developed in this section corresponds to concepts exposed traditionally in calculus courses. The contribution presented in this section consists of consolidating the abstract process in the procedure of limit calculus, making it evident that when on a curve, through its vector representation, measurements of the change are obtained, not only the initial point of the curve is reached, but also inherent and remaining characteristics of the geometric object appear, in this backward movement of the $t$ parameter, the orientation of the curve at a particular point appears. The unit magnitude tangent vector or unit tangent vector is determined by dividing the tangent vector by the magnitude of the same tangent vector.

$$
\widehat{T}=\frac{\boldsymbol{r}^{\prime}(t)}{\left\|\boldsymbol{r}^{\prime}(t)\right\|}
$$

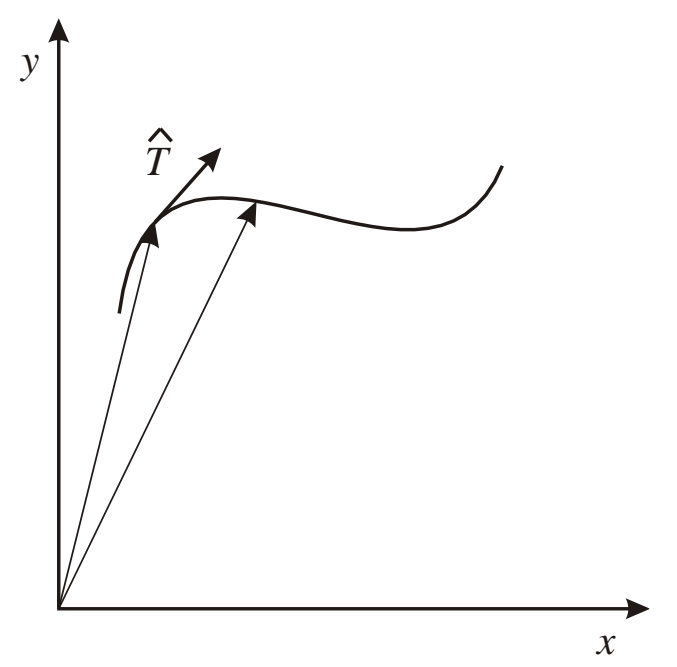

Figure 5 The unit vector tangent to a curve is of lesser magnitude than the tangent vector, however, it has a very important property and that is that its magnitude is known in advance to be constant

Another critical parameter in determining the characteristics of a curve is the normal vector. This other vector, as it can be seen, is also inherent to the curve, that is, it is another element associated with the geometric object.
Consider Figure 6, which shows a $\mathrm{C}$ curve and the tangent vectors associated with each point of interest on the curve.

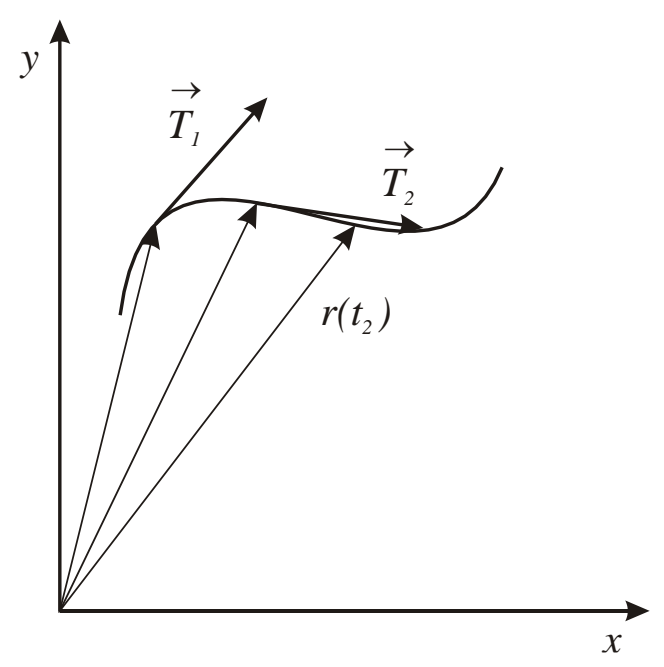

Figure 6 The $\mathrm{C}$ curve will have a tangent vector at each point of the curve. Two contiguous points of interest are shown in this figure

Figure 7 illustrates the variations of the vector $\mathrm{T}$, as the parameter $\mathrm{t}$ is traversed, in the decreasing direction.

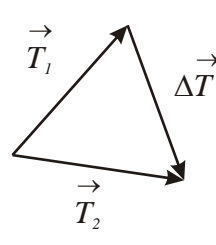

a)

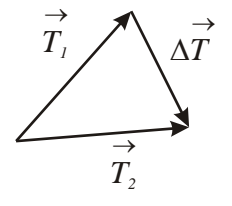

b)

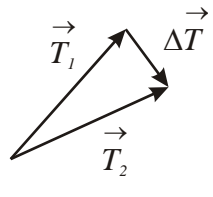

c)

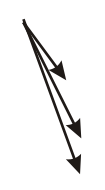

d)

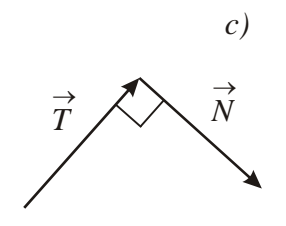

e)
Figure 7 This figure illustrates the changes made by the vector $\Delta \mathrm{T}$, as the parameter $\mathrm{t}$ returns to the point corresponding to $\mathrm{T} 1$

At the limit of the variations of the tangent vector $\Delta \boldsymbol{T}$, a vector appears that is perpendicular to the tangent vector, which is $N=$ $\vec{N}$. In Figures 7 a) to 7 c) it is observed how in the process of the limit $\Delta t \rightarrow 0$, or $t_{2} \rightarrow t_{1}$, the vector $\Delta \boldsymbol{T}$ is rotating, see Figure $7 \mathrm{~d}$ ), and is changing its magnitude, this information is manifested in the existence of the vector $\mathrm{N}$, that is perpendicular to the vector $\mathrm{T}$. 
Although the magnitude of the vector $\mathrm{T}$ is diminishing, the information regarding the change of direction and magnitude is represented by the vector $\mathrm{N}$, that is, the vector $\mathrm{N}$ represents the changes of the vector $\mathrm{T}$, and has the characteristic of being a perpendicular vector to the tangent vector, this normal vector, is also a parameter of the curve $\mathrm{C}$.

The analytical expression corresponding to the process of evaluating the changes illustrated in Figure 7, is expressed with the following calculation,

$\vec{N}=\lim _{\Delta t \rightarrow 0} \frac{\Delta \vec{T}}{\Delta t}=\frac{d \vec{T}}{d t}=\vec{T}^{\prime}(t)$

The previous formalization of the meaning of the normal vector $\mathrm{N}$, on the measure of the change of the tangent vector $\mathrm{T}$, leads to the intuition that also the normal vector $\mathrm{N}$ has been represented in terms of the proper curve, i.e., from equation (1), it is concluded that

$\vec{N}=\vec{T}^{\prime}(t)=\frac{d \boldsymbol{r}^{\prime}(t)}{d t}=\boldsymbol{r}^{\prime \prime}(t)$

and in turn, the unit normal vector is determined by the expression

$\widehat{N}=\frac{\vec{T}^{\prime}(t)}{\left\|\vec{T}^{\prime}(t)\right\|}=\frac{r^{\prime \prime}(t)}{\left\|r^{\prime \prime}(t)\right\|}$

Again, it is important to note that the meaning of this information is not made explicit in the general literature, so the student or teacher may be left with doubts regarding the nature of the derivative of a vector. Figure 8 illustrates the geometric relationship between the curve $\mathrm{C}$, the tangent vector $\mathrm{T}$, and the normal vector $\mathrm{N}$.

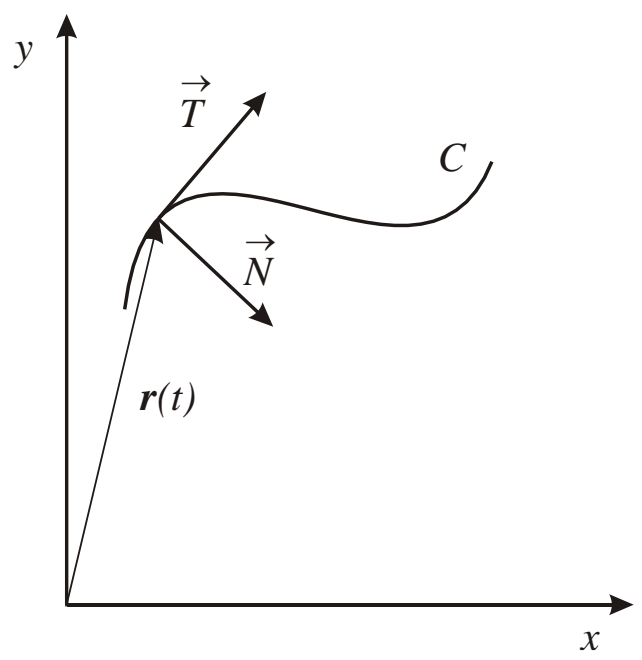

Figure 8 Geometric relationship between the curve C, its tangent vector $\mathrm{T}$ and the normal vector $\mathrm{N}$

\section{Curvature of a flat curve}

Studying and analyzing the ideas proposed in the conventional literature, for example, Anton and Leithold, among others, the curvature is obtained by quantifying the changes of the tangent vector along a curve. This is the fundamental idea to define what curvature is; however, it is quite abstract.

To use this definition of curvature, we use the definition of arc length, which allows us to obtain a tangible representation of the curve, because it can be illustrated and even measured. It is possible to determine the curvature using functional representations, but the idea of arc length allows referring to the curve as a geometric element.

The arc length, as it will be seen, corresponds with a representation of the curve $C$. This expression allows us to observe and represent the curve directly, along with its characteristics, such as the tangent vector $\mathrm{T}$ and the normal $\mathrm{N}$ and the local changes of it.

Next, we present a series of figures that illustrate the representation of a curve through the definition of arc length, that is to say, first of all, we must have a curve as the definition.

Consider the definition of arc length,

$s=\int_{t_{0}}^{t}\left\|\boldsymbol{r}^{\prime}(t)\right\| d t$

This definition can be very ambiguous for the student. Common doubts found in the classroom are the following Why derive an expression and then integrate it? What is obtained through these operations?

Next, we illustrate the meaning of these operations of calculus with several figures.

Figure 9 shows the curve $\mathrm{C}$, the position vector of a point using the vector $\mathrm{r}(\mathrm{t})$, and the tangent vector at that point, that is, $\mathrm{r}^{\prime}(\mathrm{t})$. 


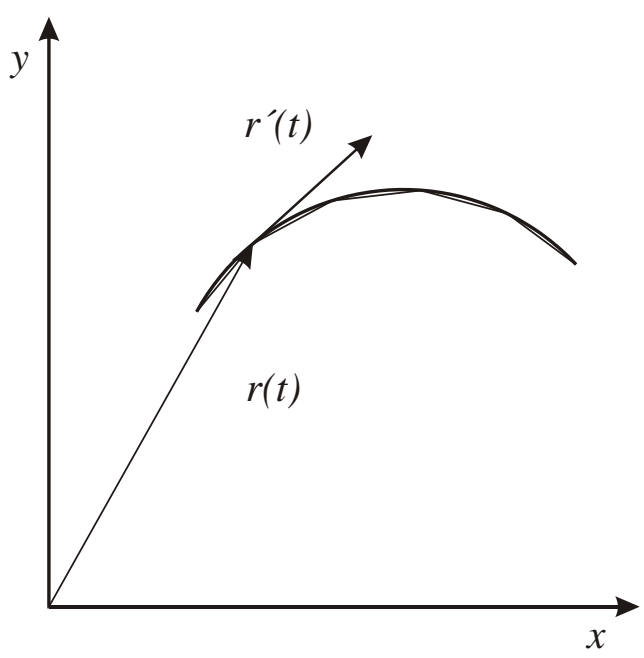

Figure 9 In a $\mathrm{C}$ curve some sectors have been determined, defined by particular points of the same curve, the tangent vector can be located in each of these points

In Figure 10, we illustrate the above curve, and locate the following vector, $\mathrm{r}^{\prime}(\mathrm{t}) \mathrm{dt}$. Note that this vector is located at the point where the tangent vector has been determined. However, when multiplied by the differential dt, this vector has the corresponding differential and vector properties; in addition, we obtain a vector and differential approximation of the curve.

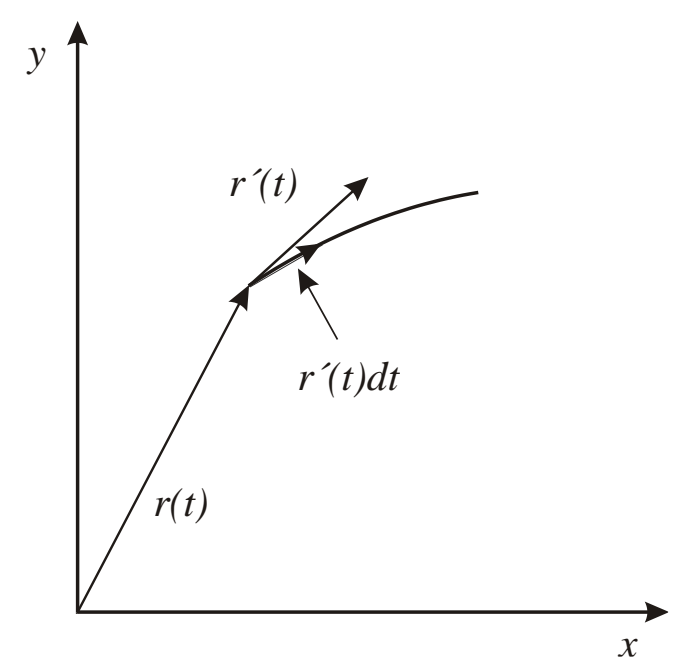

Figure 10 Construction of the $\mathrm{C}$ curve by means of the tangent vector, by multiplying the tangent vector by the differential dt, it has been possible to obtain a differential sector of the curve in vector form

The next step to obtain a representation of the curve is to eliminate the vectorial characteristic, because the curve as such is quantified in a scalar way, this is obtained by applying the absolute value function, $\left\|\boldsymbol{r}^{\prime}(t) d t\right\|$, the above is illustrated in Figure 11.

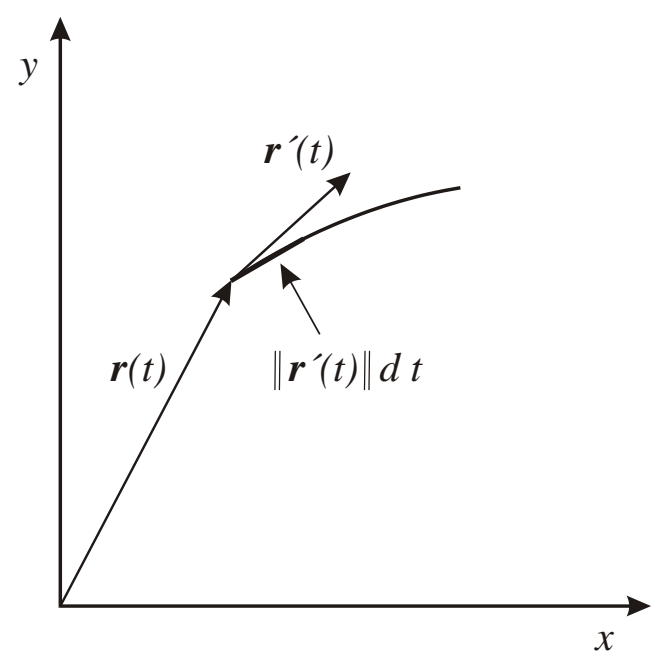

Figure 11 When the absolute value of the vector that resembles a sector of the curve is obtained, a scalar sector of the curve is obtained

Finally, the process of integration of the scalar elements, obtained from the definition of the tangent, allows obtaining the representation of the searched curve, keeping its intrinsic properties.

Figure 12 shows the result of the complete process of integration of the elements that were considered step by step.
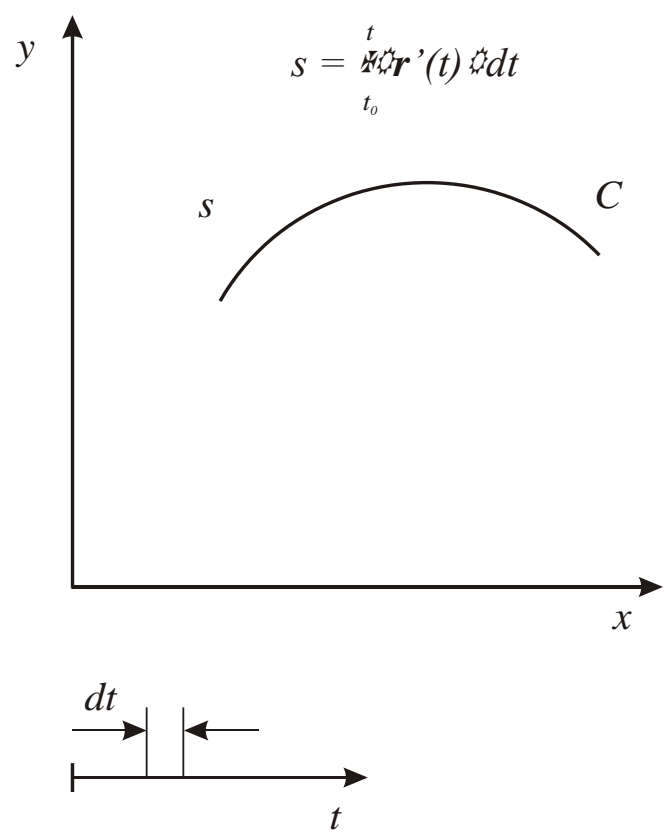

Figure 12 The differential sum of all scalar sectors allows a total representation of the curve

We have obtained a curve the characteristics of which now are referred on itself, that is to say, they are of intrinsic form. There is a relationship between the arc length s, and the parameter $t$, which previously was plotted in isolation from the curve, now can be observed directly to the curve and evaluate the changes, also directly.

VÁZQUEZ-GONZÁLEZ, Benjamín, JIMÉNEZ-RABIELA, Homero, BRAVO-ACOSTA, Adrian Gustavo and QUINTANA-DIAZ, María Berenice Guadalupe. Conceptual contributions to the determination of the curvature in Engineering courses. Journal-Mathematical and Quantitative Methods. 2019 
However, to operate on the curve, the relationship between the $\mathrm{t}$ parameter and the curve expressed by s will continue to be used. This expression is as follows,

$$
\frac{d s}{d t}=\left\|\boldsymbol{r}^{\prime}(t)\right\|
$$

Which has been derived from equation (6).

The expressions and explanations developed above will allow the concept of curvature to be clearly defined. The fundamental ideas about the existence of a circle that coincides at a point on a curve originate from the theorem of analytical geometry that states that it is possible to construct a circle: Three points $\mathrm{P}$, $\mathrm{Q}$, and non-aligned points of a plane curve determine a circle.

Consider the following figure with a curve and three chosen points of it; see McCleary.

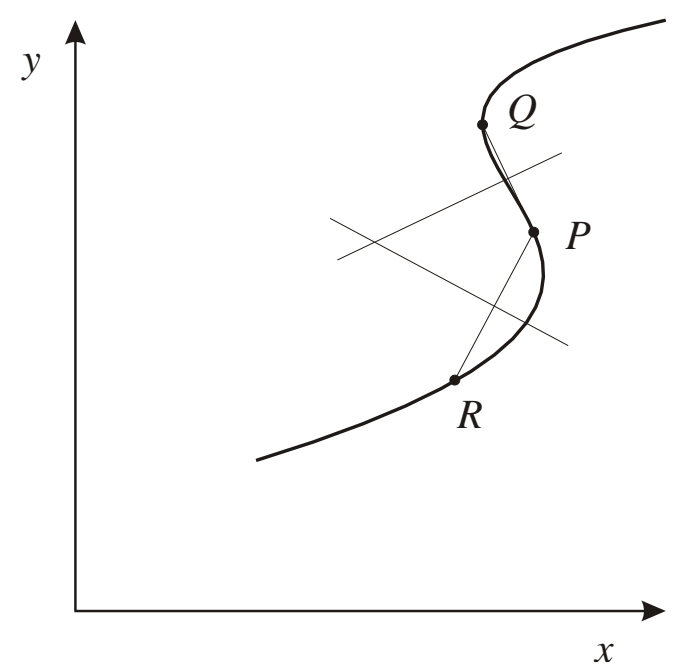

Figure 13 Determination of the center of a circle from three points belonging to the curve $\mathrm{C}$

In Figure 13, a curve appears and we draw the bisectors corresponding to the lines defined between PQ and PR. The intersection of the bisectors makes it possible to locate the center of the circle passing through the three points of the curve.

Figure 14 shows the circumference corresponding to the three points of the curve.

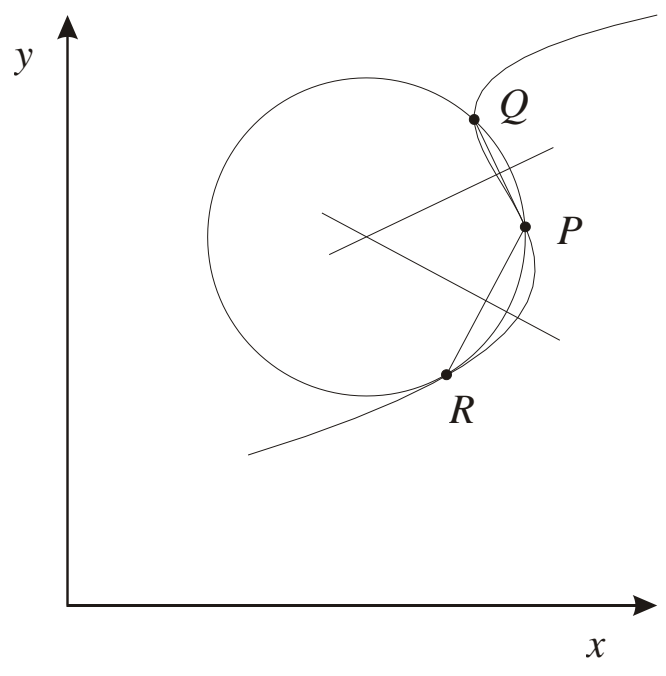

Figure 14 Construction of a circle intersecting three points of a C-curve

As the points $\mathrm{Q}$ and $\mathrm{R}$ move towards $\mathrm{P}$, we find a circle that touches the curve at point $P$.

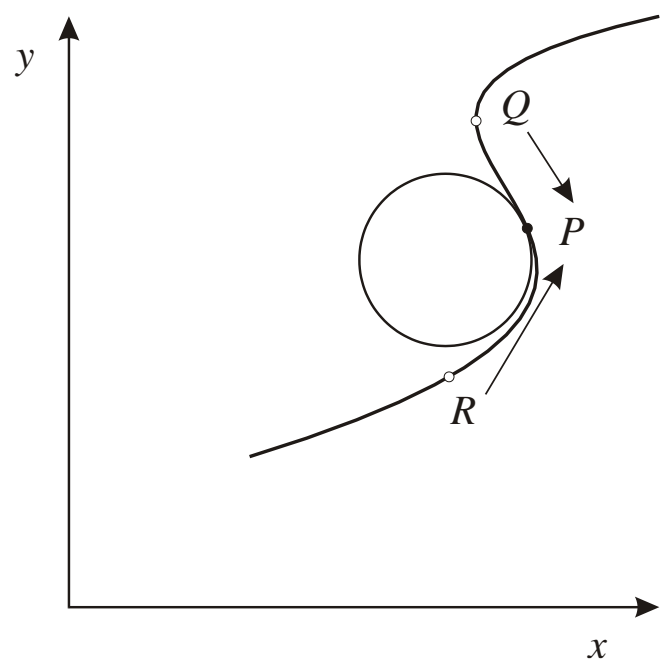

Figure 15 Determination of a circumference that coincides with the $C$ curve at point $P$

These notions provide guidelines for determining the circumference that touches the curve at a point on the curve. Curvature can be conceptualized in several ways, for example, by considering common geometric shapes.

Note that curvature is being defined in terms of the circumference that touches the curve at a point, so curvature is a measure of the curve that is compared to a circumference.

To intuitively approach the concept of curvature, through the circumference, consider the following figure. 


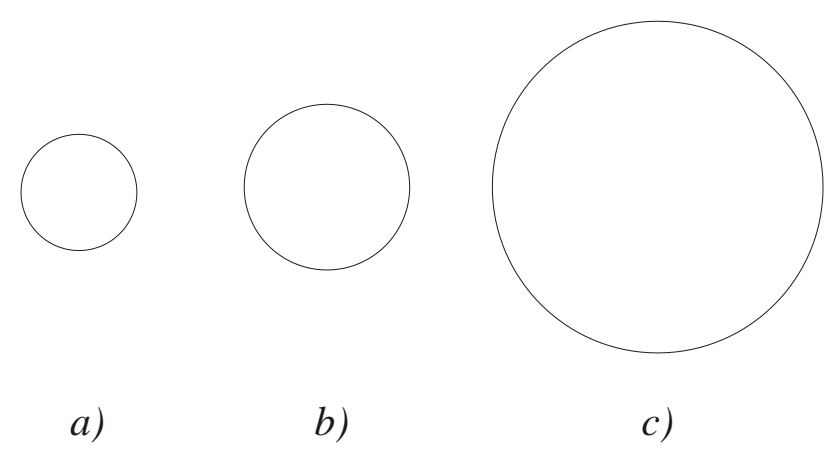

Figure 16 Three circles of different radii illustrate the concept of curvature, the larger the radius of the circle, the smaller the curvature

In the case of the circles shown, the curvature of the circle a) is greater than that of b) and that of $c$ ), the curvature of $b$ ) is greater than that of c). The curvature of the circumference a) is greater than c), because it changes faster when going around the circumference. But the radius of the circumference a) is smaller than that of b) and much smaller than that of c). In this way, we can conclude that as the curvature increases, the radius decreases, therefore the curvature is the inverse of the radius.

In the case of a straight line, we can say that its curvature is zero, and its radius is infinite if compared to a circle.

In order to establish the curvature of a curve, it is necessary to use another parameter specific to the curve. A proper parameter is the straight line tangent to the curve because it corresponds to a particular point on the curve.

The curvature of a curve at a point $\mathrm{P}$ can be defined as the variation of the unit vector tangent $\mathrm{T}$, with respect to the curve at that point, Davis and Snider. Again, this definition can be considered somewhat abstract because it establishes a measure of change, in this case of the tangent vector $T^{\wedge}$. However, parameters specific to the elements that accompany a curve must be used, such as the relation between the curve and its tangent vector.

It has been established how having a representation of the curve helps to directly observe the elements that characterize it, as well as their change on the same curve.

A specific shape of the path is established using the description $\mathrm{r}(\mathrm{s})$.
The path can be represented by the parameter arc length $s$. This is one of the advantages of using the arc length as a curve definition parameter since it can be derived directly. To obtain a simple representation of this variation, consider the following figure.

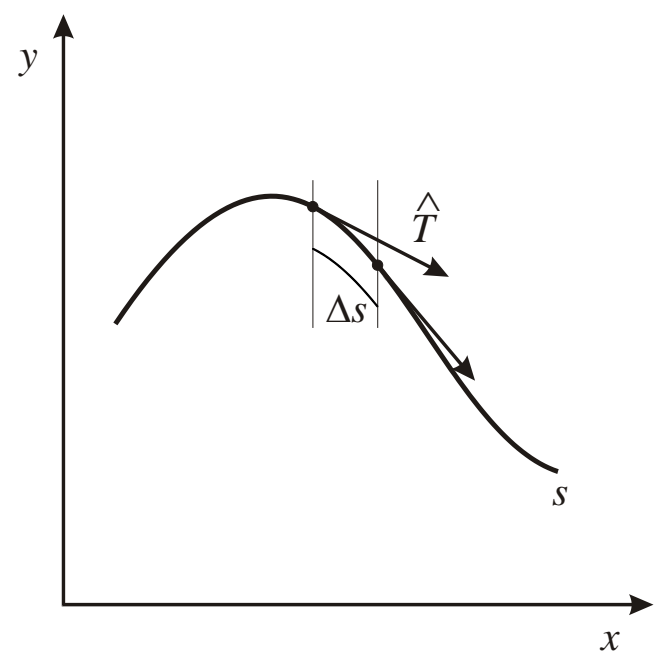

Figure 17 Identification of the parameters with which the definition of curvature can be constructed, this figure illustrates the variations of the unit tangent vector $\mathrm{T}$, as the curve develops, as arc length

In order to construct the previous definition, Figure 17 shows the basic elements with which the previous definition is constructed; however, other elements will be considered later.

Figure 17 shows two tangent vectors in two points of a curve, for simplicity, only one of them is identified. Also, a section of the curve appears, represented by its arc length $s$; in this way, the following analytical expression of the curvature can be obtained, corresponding with the previous definition.

$\kappa(s)=\left\|\lim _{\Delta s \rightarrow 0} \frac{\Delta \vec{T}}{\Delta s}\right\|=\left\|\frac{d \vec{T}}{d s}\right\|$

The next step is to obtain a description of the previous parameters, which allow determining a specific expression.

In Figure 18, there are other parameters inherent to the curve described employing the arc length.

In Figure 18 a) more information is presented, two points of the curve appear, either $\mathrm{P}$ and $\mathrm{Q}$, as well as the tangent vectors in each point, these are $\widehat{\boldsymbol{T}}(P)$ and $\widehat{\boldsymbol{T}}(Q)$, a sector of the curve appears again, let it be $\Delta s$. 


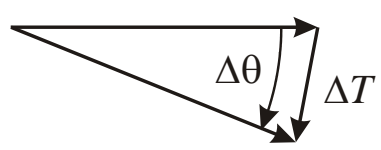

b)

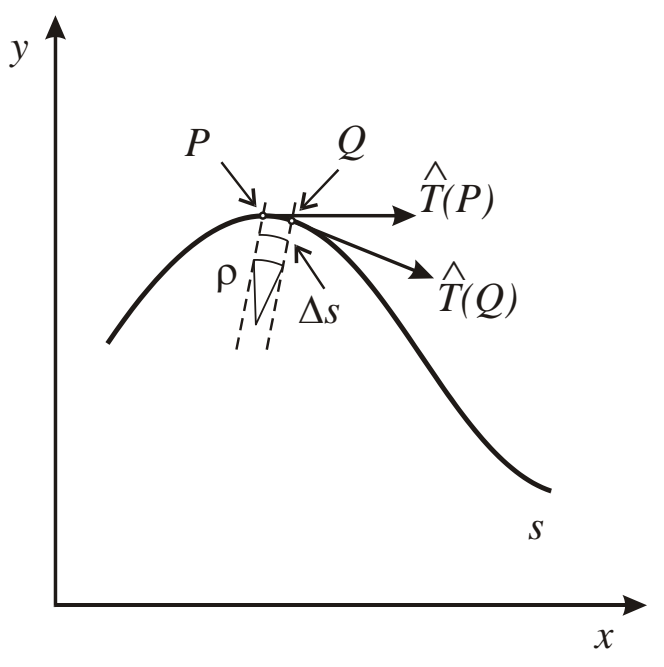

a)

Figure 18 Graphical functional relationship between two unitary tangent vectors, on a segment of the s-curve

In Figure $18 \mathrm{~b}$ ) we consider the previous tangent vectors, to compare them, they have been moved to the same point, and their deference is denoted by $\Delta T$.

As the vectors are unitary, their magnitudes are small, and therefore the magnitude of their vectorial difference can be approximated by the angle that separates them $\Delta \theta$ since, as they occupy different orientations, but are unitary, the parameter for measuring change is $\Delta \theta$, which also implies that the following proportion is met,

$$
\|\Delta \vec{T}\| \simeq\|\Delta \theta\|
$$

From the perspective of the sector $\Delta s$ of the arc length, this sector can be as a small part of a circle of radius $\rho$. It should be noted that these are the differential elements that define a circle, $\Delta s=\rho \Delta \theta$, so in this procedure, we have chosen to obtain a measure of the curvature in terms of the circle. It is possible to find other measures of curvature, for example, as stated in (14). By reconstructing equation (8) with these elements, we have the following,

$\left\|\frac{d \vec{T}}{d s}\right\|=\left\|\lim _{\Delta s \rightarrow 0} \frac{\Delta \vec{T}}{\Delta s}\right\| \simeq\left\|\lim _{\Delta s \rightarrow 0} \frac{\Delta \theta}{\Delta s}\right\|$

$\kappa(s)=\left\|\frac{d \vec{T}}{d s}\right\|=\left\|\frac{d \theta}{d s}\right\|$

Finally, $\kappa(s)=\frac{d \theta}{d s}=\frac{1}{\rho}$

This result implies that the curvature of a smooth curve in the plane can be determined and that this curvature corresponds to the curvature of a circle of radius $\rho$.

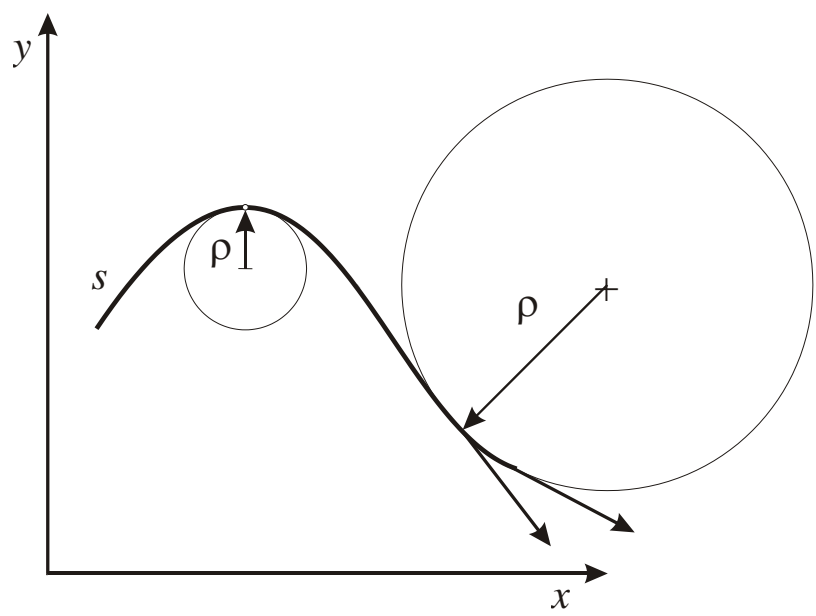

Figure 19 The osculatrix circumferences will have a radius of curvature opposite to the curvature, the greater the curvature, the smaller the radius of curvature

Figure 19 illustrates two different circumferences that an s-curve can have. In this way, at each point of interest, a circle can be located. This circle is called the osculating circle. The sharper the curve, the more closed it is, consequently the corresponding osculating circumference will have a smaller radius. However, the curvature will be greater, in relation to a less sharp curve, i.e., more open. The parameter is called the radius of curvature.

The term osculating circle was coined by Leibnitz, and is explained very well by Cruz, from whom the following paragraph is extracted: "Mathematically, the osculating circle to a curve is the one that adopts in the best way the form of the curve; the one that, when touching it, gets as close as possible to it, changing in size or position as it is more convenient in order to merge with it, at least in the point where it kisses it. Both the tangent line and the osculating circle are very similar to the curve near where they touch, but as the circle is round, it approaches it better than the line, and that is why we say that it kisses it at the moment of contact." Finally, we illustrate how the angular displacement $\Delta \theta$ the radius of the osculating circumference $\rho$. 
Consider the following example, which illustrates the relation between curvature and radius of curvature.

Three circles are shown in Figure 20, the smaller one having a radius of curvature $\rho_{1}$ and the larger having a radius of curvature $\rho_{3}$.

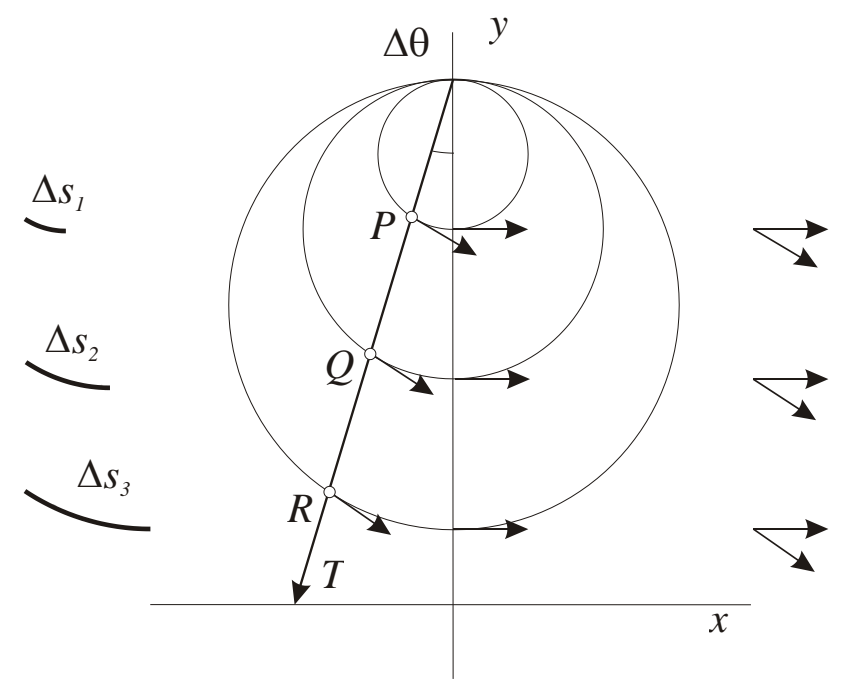

Figure 20 Example confirming the relationship between the curvature and the radius of curvature of three circles

The three circles coincide at the upper point of intersection with the vertical axis, and the line $\mathrm{T}$ originates at this point of intersection and intersects each circle at points $\mathrm{P}, \mathrm{Q}$, and $\mathrm{R}$, respectively.

In this way, the respective arcs of circumference, $\Delta s_{1}, \Delta s_{2}$ and $\Delta s_{3}$, are formed. To the left of the central figure, each of the arcs mentioned above appears separately.

Note that the angle $\Delta \theta$ at which the line $\mathrm{T}$ intersects each circle is the same, so the arcs of the circle are different, as they each have a different radius.

In each circle, two tangent vectors are illustrated. One of the tangent vectors starts at the intersection with the $y$-axis. The other tangent vector starts at the points $\mathrm{P}, \mathrm{Q}$, and $\mathrm{R}$, respectively.

To the right of the central figure are the two vectors tangent to each circle, located at the same point. The angle between the three pairs of tangent vectors remains the same. The respective curvature of each circumference is

$\kappa_{1}=\frac{d \theta}{d s_{1}}=\frac{1}{\rho_{1}}$ $\kappa_{2}=\frac{d \theta}{d s_{2}}=\frac{1}{\rho_{2}}$

$\kappa_{3}=\frac{d \theta}{d s_{3}}=\frac{1}{\rho_{3}}$

when $\Delta s \rightarrow 0$, which corresponds to the definition of curvature, according to equation (10). It is then concluded that the following relation is fulfilled,

$\kappa_{1}>\kappa_{2}>\kappa_{3}$

That is, the curvature of the smaller circumference is greater than that of the intermediate circumference and correspondingly, the curvature of the intermediate circumference is greater than the third circumference.

Therefore, it is true that

$\frac{1}{\rho_{1}}>\frac{1}{\rho_{2}}>\frac{1}{\rho_{3}}$

Or else

$\rho_{1}<\rho_{2}<\rho_{3}$

Which is the first intuitive premise to build the definition of curvature and which was illustrated through this example, that is, the more closed (acute) a curve is, the greater the curvature.

\section{Acknowledgement}

The authors are grateful for the institutional support from the Universidad Autónoma Metropolitana and the Unidad Azcapotzalco, in particular, from the Basic Sciences and Engineering Division and the Department of Energy.

\section{Conclusions}

In this work, we developed extensive definitions that have a certain degree of abstraction from the existing literature, to access the concept of curvature of a flat cure. The first important concept in the construction of the curvature is that of the length of the arch. The arc length is a graphic expression of the curve, rather than a functional parameter. It allows direct observation of the characteristics of the curve, without associating it with an external parameter.

VÁZQUEZ-GONZÁLEZ, Benjamín, JIMÉNEZ-RABIELA, Homero, BRAVO-ACOSTA, Adrian Gustavo and QUINTANA-DIAZ, María Berenice Guadalupe. Conceptual contributions to the determination of the curvature in Engineering courses. Journal-Mathematical and Quantitative Methods. 2019 
Thus, the concept of intrinsic coordinates appears. From the graphic expression, without the need to resort to an external parameter, it is possible to identify the variations of the tangent vector and obtain a measure of its curvature. The definition of curvature is based on the geometry of the circumference, which is not evident in traditional formulations. It is necessary to make explicit that we use the differential definition of the circumference to make it coincide with the variations of a curve.

Once this construction procedure is understood, it will be possible to define techniques based on the ideas of the precursors, to formulate new types of curvature.

\section{References}

Byras y Synder. (1978). Mecánica de Cuerpos Deformables. México: Representaciones y Servicios de Ingeniería, S. A.

P.A. Kralchevsky and K. Nagayama. (2001), Particles at Fluid Interfaces and Membranes Vol. 10.: Amsterdam: Elsevier.

Canoglu, M. C., Aksoy, H., \& Ercanoglu, M. (2019). Integrated approach for determining spatio-temporal variations in the hydrodynamic factors as a contributing parameter in landslide susceptibility assessments. Bulletin of Engineering Geology and the Environment, 78(5), 3159-3174.

J. E. Shigley y J. J. Uicker. (1980). Theory of Machines and Mechanisms. USA: McGrawHill.

Michael Griffis. (2003). A study of curvature for single point contact. Mechanism and Machine Theory, 38, 1391-1411.

Tenbergen, B., \& Daun, M. (2019, January). Industry Projects in Requirements Engineering Education: Application in a University Course in the US and Comparison with Germany. In Proceedings of the 52nd Hawaii International Conference on System Sciences.

Kaiser, G. (2017). Proceedings of the 13th International Congress on Mathematical Education. Germany: Springer International Publishing
Gual-Arnau, X., Ibáñez Gual, M., \& Monterde, J. (2017). Curvature approximation from parabolic sectors. Image Analysis \& Stereology, 36(3), 233-241.

Guha, M. (2013), Gaither's Dictionary of Scientific Quotations: A Collection of Approximately 27,000 Quotations Pertaining to Archaeology, Architecture, Astronomy, Biology, Botany, Chemistry, Cosmology, Darwinism, Engineering, Geology, Mathematics, Medicine, Nature, Nursing, Paleontology, Philosophy, Physics, Probability, Science, Statistics, Technology, Theory, Universe and Zoology (2nd ed.). Texas: Springer.

Arthur W. Adamson and Alice P. Gast. (1997), Physical Chemistry of Surfaces. USA: John Wiley \& Sons, Inc.

Sebastián Montiel y Antonio Ros, Curves and Surfaces, Graduate Studies in Mathematics Volume 69. American Mathematical Society Providence, Rhode Island

Sael Cruz. (2009), Palabrijes el placer de la lengua. Universidad Autónoma de la Ciudad de México, Número 4, 2-3.

Gabriele Bardini, Gian Mario Gianella, On the evolution of the idea of curvature, from Newton to Gauss' theorema egregium, Applied Mathematical Sciences, Vol. 8, 2014, no. 167, 8361-8376.

Howard Anton, Irl Bivens, Stephen Davis. (2012) Calculus early trasecendentals. USA: Howard, John Wiley \& Sons, Inc. 10th

Louis Leithold El Cálculo. (1998) México: Oxford University Press - Harla Mexico, S.A. de C.V. 7th Ed.

John MCcleary. (2013). Geometry from a Differentiable Viewpoint, Cambridge University Press.

Harry F. Davis, Artur David Snider. (1979). Introduction to Vector Analysis Fourth edition Allyn and Bacon, Inc. 